\title{
ON THE JOINT NUMERICAL STATUS AND TENSOR PRODUCTS
}

\section{RAM VERMA and LOKENATH DEBNATH}

\author{
Department of Mathematics \\ University of Central Florida \\ Orlando, Florida 32816
}

(Received September 10, 1989 and in revised form December 20, 1989)

\begin{abstract}
We prove a result on the joint numerical status of the bounded Hilbert space operators on the tensor products. The result seems to have nice applications in the multiparameter spectral theory.
\end{abstract}

KEY WORDS AND PHRASES. Joint numerical status, Hilbert tensor product, Multiparameter spectral theory, Joint Numerical range, Maximal numerical range. 1980 AMS SUBJECT CLASSIFICATION CODE. $47 \mathrm{~A}$.

\section{INTRODUCTION AND DEFINITIONS.}

In a recent paper Buoni and Wadhwa [1] introduced the concept of the joint numerical status, and have shown that the joint numerical status of commuting normal operators equals the closure of their joint numerical range. Based upon the elegant work of Stampf11 [2] on the norm of the inner derivation acting on the Banach algebra of all bounded linear operators on a Hilbert space, Fong [3] introduced the concept of essential maximum numerical range to derive the norm of an inner derivation on the Calkin algebra, and proved several interesting results. The results of Fong seem to hold for the case of the joint maximum numerical status as well.

On the other hand, Dash [4] has proved that the joint numerical range of the tensor product of operators is equal to the Cartesian product of their numerical ranges. Motivated by the work of Dash, we shall prove an analogous result for the joint numerical status of the tensor product of operators. It seems that results obtained in this paper have nice applications in the multiparameter spectral theory [5].

Let $H$ be a complex Hilbert space, and let $L(H)$ denote the algebra of all bounded linear operators on $\mathrm{H}$. The algebra $\mathrm{L}(\mathrm{H})$ is a Banach space with respect to an operator norm (See Debnath and Mikusinski [6]). In the sequel, we need the following definitions:

DEFINITION 1.1. For $A=\left(A_{1}, \ldots A_{n}\right) \in L(H)^{n}$, the joint numerical range of $A$ is denoted by $W(A)$ and defined by

$$
W(A)=\left\{\left(\left\langle A_{1} x, x\right\rangle, \ldots,\left\langle A_{n} x, x\right\rangle\right):\|x\|=1, x \varepsilon H\right\} .
$$


For the details about the joint numerical ranges, see references [1], [7] and [8].

DEFINITION 1.2. Let $C *(L(H))$ denote the $C *$-algebra generated by $I$ and $A_{1}, \ldots, A_{n}$ Then, for $A=\left(A_{1}, \ldots, A_{n}\right) \varepsilon C \star(L(H))$, the joint numerical status of $A$ is denoted by $S(A)$ and defined by

$$
S(A)=\left\{\left(f\left(A_{1}\right), \ldots, f\left(A_{n}\right)\right): f(I)=\|f\|=1\right\}
$$

where $f$ is a state on $C *(L(H)$.

We note that

$$
s\left(A_{1}, \ldots, A_{n}\right) \subseteq S\left(A_{1}\right) \times \ldots \times s\left(A_{n}\right)
$$

DEFINITION 1.3. Let $H_{1}, \ldots, H_{n}$ be complex Hilbert spaces with inner products $\langle,\rangle_{1}, \ldots,\langle,\rangle_{n}$, respectively. Let $\hat{H}=H_{1} \hat{\theta} \ldots \hat{\theta} H_{n}$ denote the Hilbert space completion of $H=H_{1} \ldots H_{n}$ with respect to inner product $\langle$, $\rangle$ which is defined by

$$
\langle x, y\rangle=\left\langle x_{1}, y_{1}\right\rangle_{1} \ldots\left\langle x_{n}, y_{n}\right\rangle_{n},
$$

for every $x, y \in H_{1} \odot \ldots H_{n} \cdot$

DEFINITION 1.4. For the operators $A_{j} \in L\left(H_{j}\right)$, the tensor product operators, $A_{j}^{-}(1<j<n)$ is defined

$$
A_{j}=I_{1} \cdots I_{j-1} \odot A_{j} \otimes I_{j+1} \odot \ldots I_{n},
$$

where $I_{j}$ is the identity operator on $\mathrm{H}_{j}$.

Clearly, $A^{\ominus}=\left(A_{1}^{0}, \ldots, A_{n}^{0}\right)$ is a commuting system of operators on $\hat{H}$.

2. A MAIN RESUlt ON THE JOINT NUMERICAL STATUS.

We now prove the following result on the foint numerical status:

THEOREM 2.1. Let $A^{\ominus}=\left(A_{1}^{0}, \ldots, A_{n}^{\theta}\right) \varepsilon C^{*}(L(\hat{H}))$. Then

$$
s\left(A_{1}^{0}, \ldots, A_{n}^{0}\right)=s\left(A_{1}\right) \times \ldots \times s\left(A_{n}\right)
$$

PROOF. In order to prove the inclusion

$$
S\left(A_{1}^{0}, \ldots, A_{n}^{0}\right) \subseteq S\left(A_{1}\right) \times \ldots \times S\left(A_{n}\right)
$$

we first prove that $S\left(A_{j}\right)=S\left(A_{j}\right)(j=1, \ldots, n)$. Since this follows by induction from the case of $n=2$, we first show that $S\left(A_{1} I_{2}\right)=S\left(A_{1}\right)$. 
If $\lambda \in S\left(A_{1}\right)$, then there exists a state f for $A_{1}$ on $C *\left(L\left(H_{1}\right)\right)$ such that $f\left(A_{1}\right)=\lambda$. Let $g$ be any state on $C^{*}\left(L\left(H_{2}\right)\right)$. Then

$$
(f \circ g)\left(A_{1} \odot I_{2}\right)=f\left(A_{1}\right) \cdot g\left(I_{2}\right)=f\left(A_{1}\right)
$$

This implies that $\mathrm{f} g$ is a state for $A_{1} \mathrm{I}_{2}$ on $\mathrm{C} *\left(\mathrm{~L}\left(\mathrm{H}_{1} \hat{\mathrm{H}}_{2}\right)\right)$, and $\lambda \varepsilon \mathrm{S}\left(\mathrm{A}_{1} \mathrm{I}_{2}\right)$.

Thus

$$
S\left(A_{1}\right) \subseteq S\left(A_{1} \odot I_{2}\right)
$$

Conversely, $\lambda \in S\left(A_{1} \otimes I_{2}\right)$, then there exists a state $h$ for $A_{1} I_{2}$ such that $h\left(A_{1} \bigcirc I_{2}\right)=\lambda$.

We next define a functional $f$ for each $A \varepsilon C \star\left(L\left(H_{1}\right)\right)$ such that $f(A)=h\left(A I_{2}\right)$. Then $f\left(I_{1}\right)=h\left(I_{1} I_{2}\right)=1$,

$$
\begin{aligned}
\|\mathrm{f}\|=\sup _{\|\mathrm{A}\|<1}|\mathrm{f}(\mathrm{A})| & =\sup _{\left\|\mathrm{A} \mathrm{I}_{2}\right\|<1}\left|\mathrm{~h}\left(\mathrm{~A} \theta \mathrm{I}_{2}\right)\right| \\
& <\|\mathrm{h}\|=1 \text {, and }\|\mathrm{f}\|=1 .
\end{aligned}
$$

Hence

$$
\lambda=h\left(A_{1} \cdot I_{2}\right)=f\left(A_{1}\right) \varepsilon S\left(A_{1}\right)
$$

This implies that $S\left(A_{1} I_{2}\right) \subseteq S\left(A_{1}\right)$. This completes the proof of this part. Similarly, we can prove that $S\left(I_{3} A_{1}\right)=S\left(A_{1}\right)$. This result combined with inclusion (1.3) establishes the theorem one way.

To prove converse part of the Theorem, let $\lambda=\left(\lambda_{1}, \ldots, \lambda_{n}\right)$ be an element of $S\left(A_{1}\right) \times \ldots \times S\left(A_{n}\right)$. Then there exists some state $f_{j}$ for $A_{j}$ such that $\lambda_{j}=f_{j}\left(A_{j}\right)$ for all $j, 1<j<n$. Now, if we set

$$
f^{\ominus}=f_{1} \otimes \ldots \otimes f_{j-1} \otimes f_{j} \otimes f_{j+1} \odot \ldots f_{n},
$$

then $f^{\theta}$ is a state on $C \star\left(L\left(H_{1} \hat{\theta} \ldots \hat{\theta}_{n}\right)\right)$, and $\lambda_{j}=f^{\theta}\left(A_{j}\right)=f_{j}\left(A_{j}\right)$ for all $j$, $1 \leqslant j \leqslant n$. This implies that $\lambda \varepsilon S\left(A_{1}^{0}, \ldots, A_{n}^{*}\right)$, and

$$
s\left(A_{1}\right) \times \ldots \times s\left(A_{n}\right) \subseteq s\left(A_{1}, \ldots, A_{n}\right)
$$

This completes the proof.

REMARK. For $A_{1}, \ldots, A_{n}$ commuting normal operators, we find the connection

$$
S\left(A_{1}^{\theta}, \ldots, A_{n}^{\theta}\right)=W\left(A_{1}^{\theta}, \ldots, A_{n}^{\theta}\right)^{-}=\operatorname{co}\left(\sigma_{\pi}\left(A_{1}^{\theta}, \ldots, A_{n}^{\theta}\right)\right),
$$


where the bar denotes the closure, and $\operatorname{co}\left(\sigma_{\pi}\left({ }^{\prime}\right)\right)$ denotes the convex hull of the joint aproximate point spectrum [1].

\section{REFERENCES}

1. BUONI, J. and WADHWA, B., On joint numerical ranges, Pacific J. Math. 77 (1978), 303-306.

2. StAMPfl, J., The norm of a derivation, Pacific J. Math 33 (1970), 737-747.

3. FONG, C., On the essential maximal numerical range, Acta_Sci. Math. 41 (1979), 307-315.

4. DASH, A.T., Tensor products and joint numerical range, Proc..Amer. Math. Soc. 40 (1973), 521-526.

5. VERMA, R., Multiparameter spectral theory of a separating operator system, Applied Math. Letters 2(4) (1989) 391-394.

6. DEBNATH, L., and MIKUSINSKI, P., Introduction to Hilbert Spaces with Applications, Academic Press, Boston 1990.

7. DASH, A.T., Joint numerical range, Glasnik Mat. $\underline{7}$ (1972), 75-81.

8. HALMOS, P., A Hilbert Space Problem Book, second edition, Springer -Verlag, New York, 1982. 


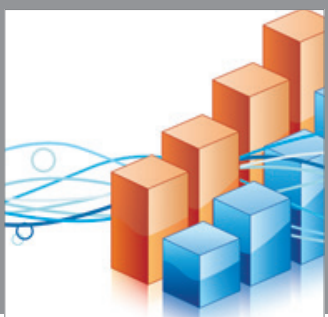

Advances in

Operations Research

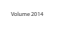

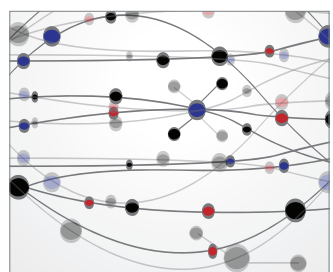

\section{The Scientific} World Journal
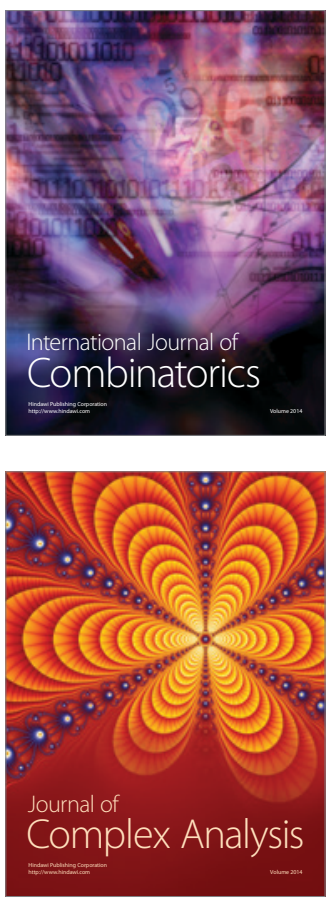

International Journal of

Mathematics and

Mathematical

Sciences
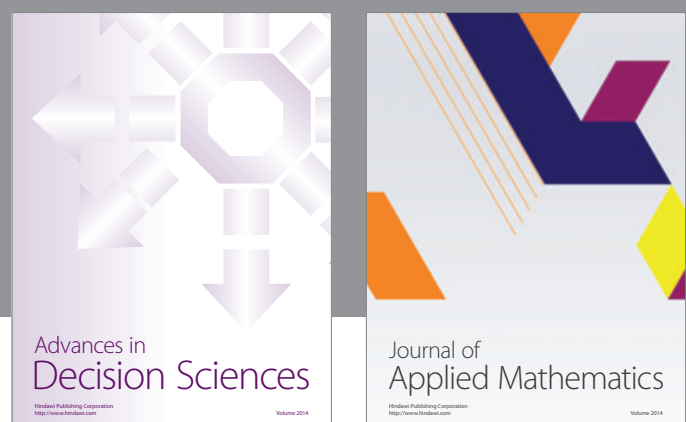

Journal of

Applied Mathematics
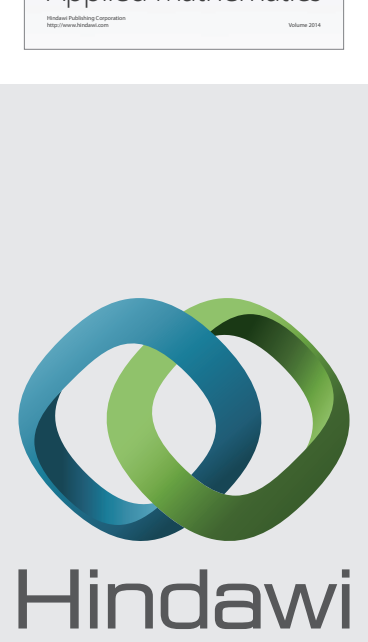

Submit your manuscripts at http://www.hindawi.com
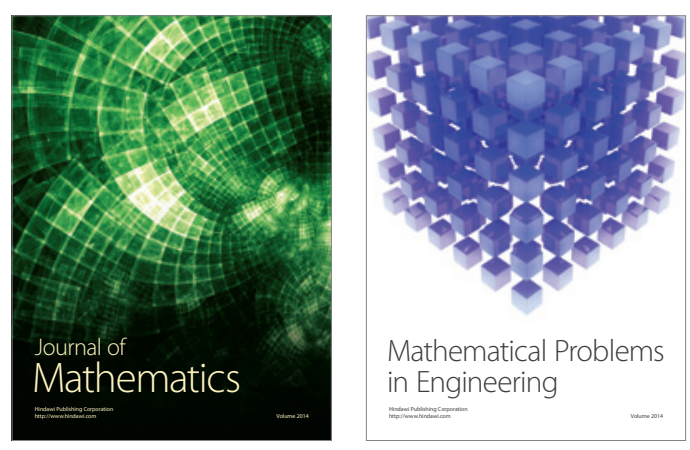

Mathematical Problems in Engineering
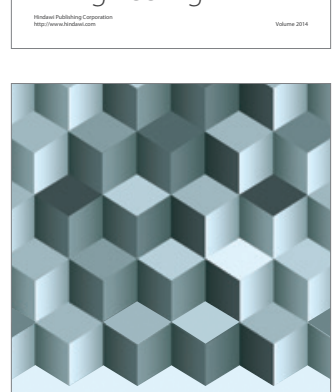

Journal of

Function Spaces
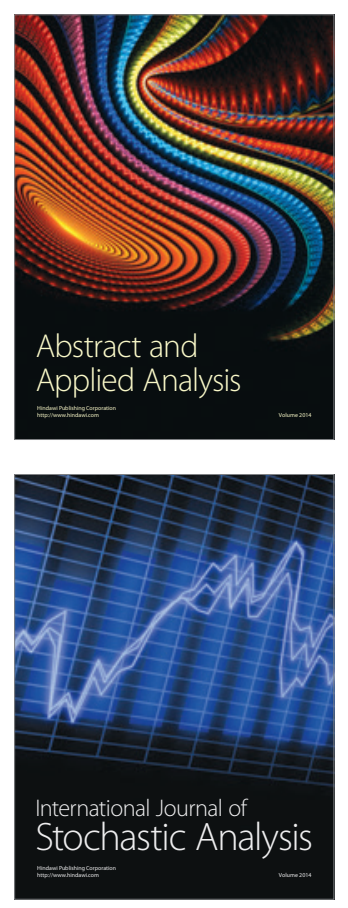

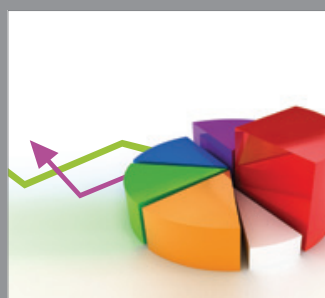

ournal of

Probability and Statistics

Promensencen
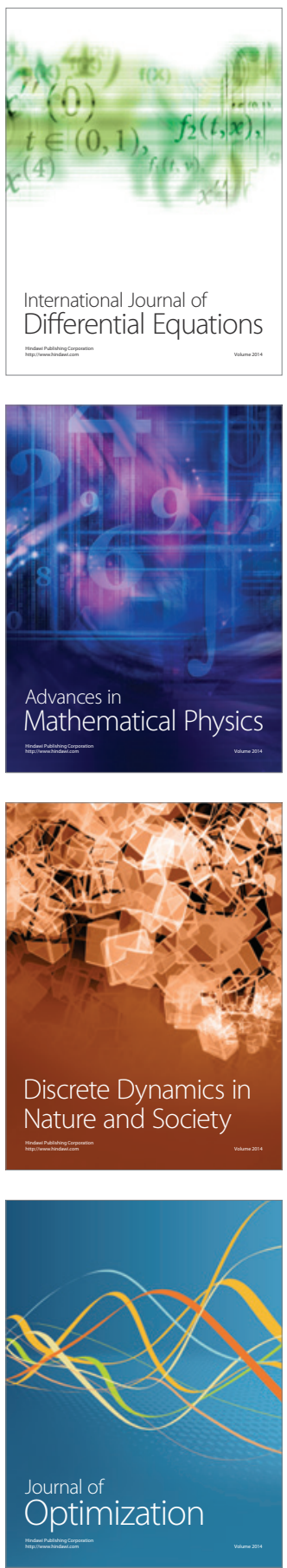